\title{
Neutrino-triggered target-of-opportunity programs in IceCube
}

\author{
The IceCube Collaboration ${ }^{\dagger}$, \\ $\dagger$ http://icecube.wisc.edu/collaboration/authors/icrc15_icecube \\ E-mail: Dariusz.Gora@desy.de
}

\begin{abstract}
IceCube is capable of monitoring the whole sky continuously, while optical and high energy photon telescopes have limited fields of view and are not likely to observe a potential neutrino-flaring source at the time such neutrinos are recorded. The use of neutrino-triggered alerts thus aims at increasing the availability of simultaneous multi-messenger data, which can increase the discovery potential as well as constrain the phenomenological interpretation of the high energy emission of selected source classes. The requirements of a fast and stable online analysis of potential neutrino signals and its operation will be discussed. The status and the recent improvements of a neutrinotriggered program in IceCube are described. The currently running systems generate real-time alerts based on multiplets of neutrinos occurring close in time and space, and these alerts are received for follow-up observations by various instruments, ranging from optical (PTF) and X-ray (Swift) to gamma-ray (H.E.S.S., MAGIC and VERITAS). The possibility in the near future to additionally send alerts based on single high energy neutrino events of likely astrophysical origin will also be discussed.
\end{abstract}

\footnotetext{
Corresponding authors: Dariusz Góra ${ }^{1 *}$, Elisa Bernardini ${ }^{1,2}$, Marek Kowalski ${ }^{1,2}$, Markus Voge ${ }^{3}$, Alexander Stasik ${ }^{2}$, Thomas Kintscher ${ }^{1}$

${ }^{1}$ Institut für Physik, Humboldt Universität, Newtonstr. 15, D-12489 Berlin, Germany

${ }^{2}$ Deutsches Elektronen-Synchrotron (DESY), Platanenallee 6, D-15735 Zeuthen, Germany

${ }^{3}$ Physikalisches Institut, Universität Bonn, Nussallee 12, D-53115 Bonn, Germany
}

The 34th International Cosmic Ray Conference,

30 July- 6 August, 2015

The Hague, The Netherlands

\footnotetext{
*Speaker.
} 


\section{Introduction}

Neutrinos have long been anticipated to help answer some fundamental questions in astrophysics like the mystery of the source of the cosmic rays (for a general discussion see [1]). For neutrinos in the $\mathrm{TeV}$ range, prime source candidates are Galactic supernova remnants [2]. Neutrinos in the PeV range and above are suspected to be produced by Active Galactic Nuclei (AGN) and Gamma Ray Bursts (GRB) with many AGN models predicting a significant neutrino flux $[3,4,5]$. Recently, the IceCube Collaboration has reported the very first observation of a cosmic diffuse neutrino flux which lies in the $100 \mathrm{TeV}$ to $\mathrm{PeV}$ range [6]. Individual sources, however, have not been identified. While many astrophysical sources of origin have been suggested, there is yet not enough information to narrow down the possibilities to any particular source.

The detection of cosmic neutrinos by high-energy neutrino telescopes is very challenging due to the small neutrino interaction cross-section and because of a large background of atmospheric neutrinos. Thus, simultaneous measurements using neutrino and electromagnetic observations (the so-called "multi-messenger" approach) can increase the chance to discover the first neutrino signals from astrophysical source by reducing the trial factor penalty arising from the observation of multiple sky regions over different time periods.

For slower transient sources like AGNs (time scale of weeks) which manifest large time variations in the emitted electromagnetic radiation, the signal-to-noise ratio can be increased by searching for periods of enhanced neutrino emission (a time-dependent search). Of special interest is the relation of these periods of enhanced neutrino emission with periods of strong high-energy $\gamma$-ray emission. However, as Imaging Air Cherenkov Telescopes (IACTs) such as MAGIC [7] or VERITAS [8] have a small field-of-view and are not continuously operated such correlation studies are not always possible after the flare. Therefore it is desirable to ensure the availability of simultaneous neutrino and high-energy $\gamma$-ray data for periods of interests. This is achieved by an online neutrino flare search that alerts a partner IACT experiment when an elevated rate of neutrino events from the direction of a source candidate is detected. Such a Neutrino Triggered Target of Opportunity program $(\mathrm{NToO})$ using a list of pre-defined sources was developed already in 2006 using the AMANDA neutrino telescope to initiate quasi-simultaneous gamma-ray follow-up (GFU) observations by MAGIC [9]. The refined and enhanced implementation of GFU, using the data from IceCube neutrino detector to sent alerts to $\gamma$-ray telescopes MAGIC and VERITAS, was started on March, 2012 [10].

Similarly, one can conduct a search for neutrinos from short transient sources (time scale of 100 seconds), such as GRBs (see e.g. [11]) and core-collapse Supernovae (SNe) (see e.g. [12]). These sources are most accessible in X-ray and optical wavelengths, where one can observe the GRB afterglow or the rising SN light curve, respectively. As for IACTs, the field of view and observation time of X-ray and optical telescopes is limited. Since identification of a GRB or SN is only possible within a certain time range (few hours after a GRB and few weeks after a SN explosion), it is important to obtain electro-magnetic data within these time frames. Therefore, another NToO program targeting optical (OFU) and X-ray (XFU) follow-up of short neutrino transients has been developed as well since 2008 [10, 13]. Upon multiplets (at least 2) of neutrinos within 100 seconds and $3.5^{\circ}$ angular separation (searching anywhere in the sky), alerts are sent to ROTSE [14], PTF [15] for optical and Swift [16] for X-ray follow-up, depending on the multiplet's significance. 


\section{Selection of Target Sources}

As already mentioned, the online search for short transient neutrino sources is mostly motivated by models of neutrinos from long durations GRBs [11] and from choked jet supernovae (SNe) [12] that are SNe hosting a mildly relativistic jet. The two source classes are related: Both are thought to host a jet, which is highly relativistic in case of long GRBs, but only mildly relativistic in case of choked jet SNe. However, the choked jet is less energetic and thus cannot penetrate the stellar envelope, making it invisible in $\gamma$-rays. The produced neutrinos at $\mathrm{TeV}$ energies can escape nevertheless and might trigger a discovery of a SN in the follow-up channels. Both sources are expected to emit a short, about $10 \mathrm{~s}$ long burst of neutrinos either 10 to $100 \mathrm{~s}$ before or at the time of the $\gamma$-ray burst, setting the natural timescale of the neutrino search. After recording the neutrino burst, follow-up observations can be used to identify the counterpart of the transient neutrino source. The $\gamma$-ray burst itself is generally too short for a follow-up to IceCube data, since 95\% of GRBs have a duration of less than $150 \mathrm{~s}$. However, there are good detection prospects using the GRB afterglow, in optical or in X-ray data. A very fast response within minutes to hours is required for this. A choked jet SN is found by detecting a shock breakout or a SN light curve in the follow-up images, slowly rising and then decaying within weeks after the neutrino burst. In addition to the transient neutrino emission within $100 \mathrm{~s}$, as discussed above, SNe can be promising sources of high-energy neutrinos, especially the class of Type IIn SNe [17]. These are core-collapse $\mathrm{SNe}$ embedded in a dense circumstellar medium (CSM) that was ejected in a pre-explosion phase. Following the explosion, the SN ejecta plow through the dense CSM and collision less shocks can form and accelerate particles, which may create high-energy neutrinos. This is comparable to a SN remnant, but on a much shorter time scale of 1 to 10 months.

Coordinated observation of gamma-rays and neutrinos might be possible for sources where charged and neutral mesons are produced simultaneously, from hadronic $\mathrm{p}-\mathrm{p}$ or $\mathrm{p}-\boldsymbol{\gamma}$ interactions. This concerns variable objects such as Blazars or Flat-Spectrum Radio Quasars (FSRQs), as well as Galactic systems like microquasars and magnetars, whose emission is interpretable with hadronic models. The chance of detection of individual AGN flares, on a time scale of days or weeks, is estimated on different predictions for the mechanism yielding the observed electromagnetic emission at high energies [3, 4]. A common feature of several models is that the class of high energy peaked BL Lacs (HBLs) is expected to be weakest in neutrinos, as compared to low energy peaked BL Lacs (LBLs) and FSRQs, if interactions of protons with ambient or self-produced radiation significantly contribute to the observed high energy gamma-ray emission. With high target matter density, the neutrino yield can be highest when the very high energy $\gamma$-ray emission is strongly attenuated by internal absorption, with cut-off values, however, being uncertain. In case of pp-dominated scenarios, conclusions are different [4], favoring LBLs to FSRQs. In all cases, the availability of simultaneous information on high energy $\gamma$-ray emission and neutrinos is crucial.

The most interesting objects as a target for GFU observations triggered by IceCube events are promising sources of $\mathrm{TeV}$ neutrinos, which are either known to exhibit a bright $\mathrm{GeV}$ flux in $\gamma$ rays and show extrapolated fluxes detectable by IACTs, or are already detected by IACTs and are variable. Thus, for GFU the selected list of AGNs from the second Fermi point-source catalog [20] combined with the lists provided by the partner experiments (currently MAGIC and VERITAS) covering the Northern hemisphere $\left(\delta>0^{\circ}\right)$ are used, see [10] for more details. 


\section{The IceCube follow-up alert issuing system}

IceCube is a cubic-kilometer neutrino detector installed in the ice at the geographic South Pole [21] between depths of $1450 \mathrm{~m}$ and $2450 \mathrm{~m}$. Detector construction started in 2005 and finished in December 2010. Neutrino reconstruction relies on the optical detection of Cherenkov radiation emitted by secondary particles produced in neutrino interactions in the surrounding ice or the nearby bedrock. For NToO programs presented here, only muon neutrino events are considered, because of the long range of the secondary muons which allows for reconstructing these events with good pointing accuracy. The pointing information relies on the secondary muon direction, which at energies above $1 \mathrm{TeV}$ differs from the original neutrino direction by less than the angular resolution of the detector. The current online data processing is running in real-time within the limited computing resources at the South Pole, capable of reconstructing and filtering the neutrinos and sending alerts to follow-up instruments with a latency of only a few minutes. The optical (OFU) and X-ray (XFU) real-time follow-up programs currently encompass three follow-up instruments: the Robotic Optical Transient Search Experiment (ROTSE) [14] ${ }^{1}$, the Palomar Transient Factory (PTF) [15] and the Swift satellite [16]. These triggered observations were supplemented with a retrospective search through the Pan-STARRS1 $3 \pi$ survey data [18]. Similarly, the GFU is sending alerts to the MAGIC and VERITAS.

The basis for the neutrino event selection is an on-line filter that searches for high-quality muon tracks. The fullsky rate of this filter is about $40 \mathrm{~Hz}$ for IceCube in its 2012/2013 configuration with 86 deployed strings. This rate is strongly dominated by atmospheric muons. In order to efficiently select neutrinos events from this sample several elaborate reconstruction algorithm have to be applied. However, as the computing resources at the South Pole are limited, this is only possible at a lower event rate. The so-called Online Level 2 Filter (Online-L2 Filter) selects events that were reconstructed as upgoing with a likelihood reconstruction that takes into account the time of arrival of the first photon at each Digital Optical Module (DOM) and the total charge recorded in that module. By requiring a good reconstruction quality the background of misreconstructed atmospheric muons is reduced. The parameters used to assess the track quality are the likelihood of the track reconstruction, the number of unscattered photons with a small time residual w.r.t. the Cherenkov cone and the distribution of these photons along the track. The reduced event rate of approximately $5 \mathrm{~Hz}$ can then be reconstructed with more time intensive reconstructions, like angular resolution estimators and likelihood fits applied to different subsets of the recorded photons. Based on these reconstructions the final event sample is selected by employing different quality cuts for OFU/XFU and GFU. The event selection results in an event rate of about $2 \mathrm{mHz}$ for GFU and $3 \mathrm{mHz}$ for OFU and a median angular resolution of $0.6^{\circ}$ for $100 \mathrm{TeV}$ and higher energies.

For OFU/XFU, in order to suppress background from atmospheric neutrinos, a multiplet of at least two neutrinos within 100 seconds and angular separation of $3.5^{\circ}$ or less is required to trigger an alert. In addition, since 2011 mid-September, a test statistic is used, providing a single parameter for selection of the most significant alerts. It was derived as the analytic maximization of a likelihood ratio following [19], for the special case of a neutrino doublet with rich signal content, see [10] for more details.

For GFU the timescale of a neutrino flare is not fixed a-priori, and the time-clustering ap-

\footnotetext{
${ }^{1}$ At the moment, there is no followup to ROTSE anymore as they went out of operation.
} 
proach that was developed for an unbiased neutrino flare search [22] looks for any time frame with a significant deviation of the number of detected neutrinos from the expected background. The simplest implementation uses a binned approach where neutrino candidates within a fixed radius around a source are regarded as possible signal event. If the significance for an evaluated event cluster exceeds a certain threshold, an alert message containing the source name, event positions, event times and the significance of the cluster is generated and the sent to partner experiments, see also [10] for more detailed description of GFU.

\section{Selected results of NToO programs}

The IceCube NToO programs like OFU/XFU/GFU have been running stably for a few years and are taking high-quality data from both IceCube and the follow-up instruments. The result will be subject of forthcoming publications [24]. Thus here, only a short status report will be given, highlighting the most important results.

For OFU/XFU, the total number of observed doublet alerts is 258 , since inauguration of the program in December 2008 until May 2014. This stands against a background expectation of $236.7 \pm 15.4$ doublets. No triplets have been observed, while the background expectation is $0.09 \pm$ 0.3 triplets. However, in March 2012, the most significant neutrino alert during the first three years of operation was issued by IceCube. In the follow-up observations performed by the PTF, a Type IIn supernova (SN) PTF12csy was found $0.2^{\circ}$ away from the neutrino alert direction, with an error radius of $0.54^{\circ}$. It has a redshift of $\mathrm{z}=0.0684$, corresponding to a luminosity distance of about $300 \mathrm{Mpc}$ and the Pan-STARRS1 survey shows that its explosion time was at least 158 days (in host galaxy rest frame) before the neutrino alert, so that a causal connection is unlikely. The a posteriori significance of the chance detection of both the neutrinos and the $\mathrm{SN}$ at any epoch is $2.2 \sigma$ within IceCube's 2011/12 data acquisition season. Also, a complementary neutrino analysis reveals no long-term signal over the course of one year. Therefore, the SN detection is considered coincidental and the neutrinos uncorrelated to the SN. However, the SN is unusual and interesting by itself: It is luminous and energetic, bearing strong resemblance to the SN IIn 2010jl, and shows signs of interaction of the SN ejecta with a dense circumstellar medium. High-energy neutrino emission is expected in models of difusive shock acceleration, but at a low, non-detectable level by IceCube experiment for this specific $\mathrm{SN}$.

For GFU, since the inauguration of the GFU program in 2012, 13 physics alerts have been sent so far (4 in 2012, 2 in 2013, 6 in 2014, 2 in 2015 ). Follow-up observations have been performed by MAGIC in three cases and by VERITAS in four cases. The most interesting alert generated on 9 November 2012 originated from the source 1150+497 (located at $\theta=139.5^{\circ}$ ). Six events observed during 4.169 days comprise the alert. The Poisson probability (pre-trial) for this observation is $-\log _{10} p=4.64$, the highest alert significance of the alerts sent during the 2012/2013 data acquisition season. The alert was forwarded to the VERITAS telescope and resulted in a follow-up observation. The VERITAS team received the alert from IceCube, but due to the poor weather and bright moonlight conditions did not allow VERITAS observations until 12 November 2012, at which point the source was visible at low elevations at the very end of the night. A further observation was made on the following night with the total exposure time of about $15 \mathrm{~min}$. No statistically significant evidence for gamma-ray emission was seen from the position of source, thus the integral upper flux limit was calculated by VERITAS. 

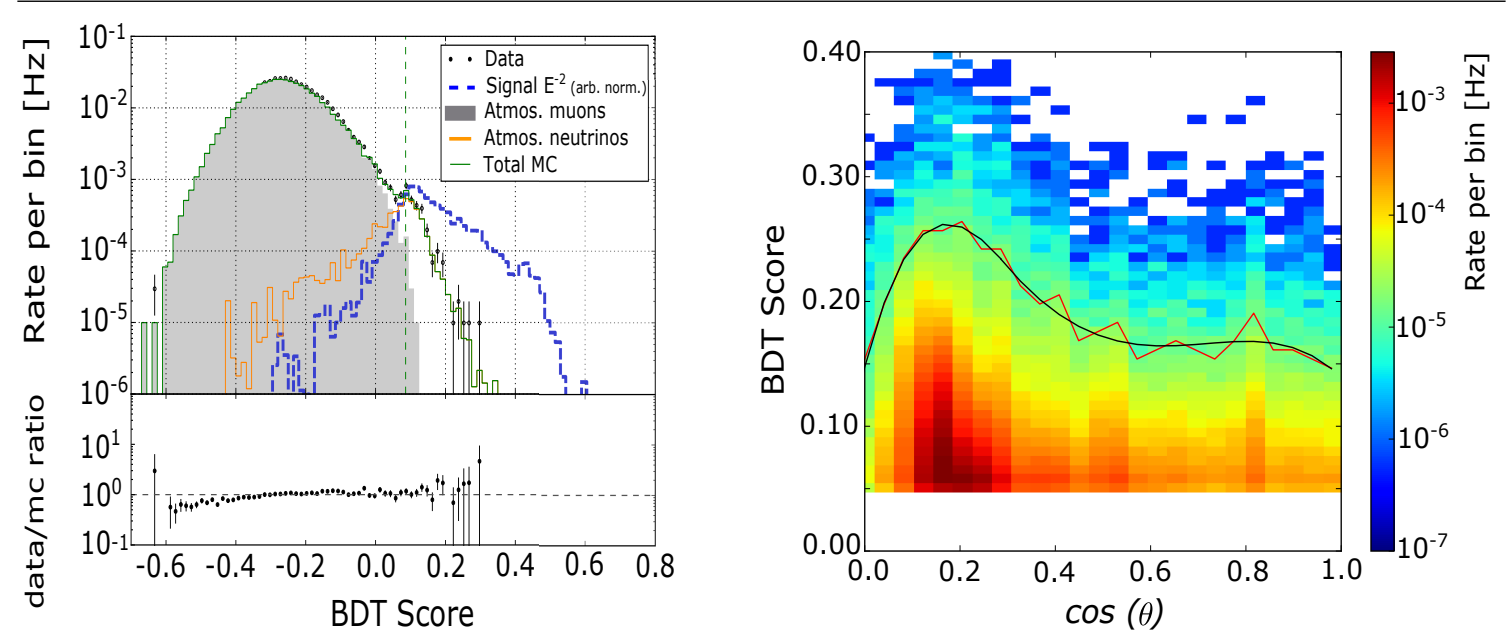

Figure 1: (A) Distribution of BDT score for the ensemble of trees trained with an $E^{-2}$ spectrum. Vertical dashed lines corresponds to optimized BDT cut (Northern hemisphere). (B) The BDT score as a function of $\cos \left(\right.$ zenith angle). The red lines keeps approximately an equal rate per zenith bin $\left(\simeq 10^{-4} \mathrm{~Hz}\right)$. A piecewise polynomial function is then fit to the red curve (black curve).

\section{Recent and upcoming improvements}

The currently deployed neutrino event selection in the GFU employs simple cuts on a number of variables that discriminate between signal neutrinos and atmospheric muon background. The cuts on these parameters have been optimized to achieve an optimal sensitivity. However, such cuts most likely do not lead to the most optimal shape in terms of signal and background separation. In order to increase the separation between signal and backgroud event distributions the Boosted Decision Trees (BDTs) [23], multi-variate learning machines, were used. In fact, for (OFU/XFU) the multi-variant selection scheme was already implemented since 2012, but it was used to cover only the Northern Hemisphere. The aim is replacing the present GFU selection by developing a new event selection, which can be used also by OFU/XFU program, will be comparable to offline point source samples and will cover the entire sky i.e. providing $4 \pi$ coverage. Here, a short description of a new BDT selection is presented, which has been implemented for 2015/2016 IceCube data acquisition season.

For a new BDT selection scheme, the multivariate cuts were based on 14 observables obtained by choosing parameters with low correlation in background but high discriminating power between signal and background. Observables specifying the geometry, the time evolution of the hit pattern as well as the quality and consistency of the various track reconstructions and the number of strings with signals are used. The BDT training was done with simulated signal events for a soft neutrino spectrum of $E^{-2.7}$ and for an $E^{-2}$ spectrum. As an example, Figure 1 depicts results of the BDT training for an $E^{-2}$ spectrum. In addition, to achieve a good result for all spectra, a new event weighting scheme was introduced, by giving each signal event a combined weight of the spectra weights (CSW): $w_{i}=\left(\frac{w_{i}^{E^{-2.7}}}{\sum_{j} w_{j}^{E^{-2.7}}}+\frac{w_{i}^{E^{-2}}}{\sum_{j} w_{j}^{E^{-2}}}+\frac{w_{i}^{E^{-1}}}{\sum_{j} w_{j}^{E^{-1}}}\right) / \sum_{j} w_{i}$. A set of off-time real data, was used for training as background. Additionally, for the simulated signal, the reconstructed track was required to be within of $5^{\circ}$ of the simulated direction in order to train BDT with only well-reconstructed events. The final selections were optimized to provide the best discovery potential for an $E^{-2}$ neutrino flux, which results in a BDT score value of 0.106 . This final cut leads to a rate of $2 \mathrm{mHz}$ for the final sample and as it shown in Table 1 to the better signal efficiency (w.r.t. to OnlineLevel-2 


\begin{tabular}{c|c|c|c|c|c} 
Cut Level & $\begin{array}{c}\text { Data rate } \\
(\mathrm{mHz})\end{array}$ & $\begin{array}{c}\text { Atm. } v_{\mu} \text { rate } \\
(\mathrm{mHz})\end{array}$ & $\begin{array}{c}\mathrm{E}^{-1} \text { Eff. } \\
(\%)\end{array}$ & $\begin{array}{c}\mathrm{E}^{-2} \text { Eff. } \\
(\%)\end{array}$ & $\begin{array}{c}\mathrm{E}^{-2.7} \text { Eff. } \\
(\%)\end{array}$ \\
\hline Simple Cuts & 2.0 & 1.9 & 79 & 69 & 54 \\
BDT $E^{-2.0}$ & 1.9 & 1.7 & 86 & 81 & 72 \\
BDT $E^{-2.7}$ & 2.7 & 2.5 & 84 & 78 & 65 \\
BDT CSW & 3.1 & 2.8 & 83 & 80 & 72
\end{tabular}

Table 1: Data, atmospheric muon, and neutrino expected background rates for different cut progression. The signal efficiency for an $E^{-2}$ neutrino spectrum with respect to the OnlineL2 Filter is also shown.

Filter efficiency) than the straight GFU cuts. The BDT-based event selection leads to the improvement in the signal efficiency of about $+11(+18) \%$ for an $E^{-2}\left(E^{-2.7}\right)$ spectrum w.r.t. the simple cuts.

The BDT selection was also used for the Southern hemisphere (zenith angle $\theta<90^{\circ}$ ). However instead of a single BDT score cut value, a zenith-dependent cut was applied in order to select a constant number of events per solid angle, as it is shown in Figure 1 (B). This zenith-dependent cut was also optimized w.r.t. sensitivity and discovery potential for an $E^{-2}$ neutrino spectrum. The optimized cut described by a polynomial fit (Figure 1 (B)) leads to the total data rate of $2.1 \mathrm{mHz}$ and average signal efficiency of about $40 \%$ (w.r.t. OnlineL2 Filter).

It was the first step in establishing the GFU, demonstrating its technical feasibility and proving that a time-dependent point source search can be run stably and reliably over long periods of time at the South Pole. Therefore a simple search technique like the binned method was implemented first. However, current offline IceCube searches for neutrino point-sources usually employ unbinned maximum likelihood methods [19] to increase the discovery potential. Thus, also for GFU such an approach was implemented, in order to calculate the alert significance by taking into account an event-by-event angular reconstruction uncertainty estimation and an energy estimation of the event. The upgrade towards the BDT-based event selection and a subsequent likelihood analysis, in case of GFU increase the sensitivity in the Northern hemisphere of about 30-40\% to a level comparable to the sensitivity obtained for the standard offline point-source analysis [25]. It also opens the possibility to observe flares on the Southern hemisphere and forward alerts to the H.E.S.S. telescope [26], with which an MoU has been established.

In previous years of operation of the OFU/XFU/GFU systems, neutrino candidate event selections, multiplet selection and alert generation all took place within the data acquisition system at South Pole. Although well-contained, it was found to be somewhat inflexible and difficult to expand. To address these shortcomings, the OFU/GFU/XFU systems are already transitioning to a new system. This system separates the neutrino candidate selection at the South Pole, and transmitting the information as a stream of single high energy events to North via rapid satellite communication channels. Followup processes in the North evaluate neutrino candidates, and generate alerts for external observatories, see [25] for more detailed description.

\section{Conclusions}

The IceCube NToO programs (OFU, XFU and GFU) have been running stably since a few years and are taking high-quality data from both IceCube and the follow-up instruments. As an example, the highest significance alert from the OFU program led to a coincidental discovery of a 
Type IIn SN, demonstrating the capability of the follow-up system to reveal transient high energy neutrino sources. Several enhancements of NToO programs have recently been implemented (i.e. BDT selections and a maximum-likelihood based significance calculation) or are planned for the near future (i.e. the extension to the Southern hemisphere). The current online alert systems are limited to the Northern hemisphere due to the enormous atmospheric muon background. Finally, changes to the online followup framework, i.e. the stream of single high energy events, are under way. Hopefully, these improvements to NToO programs will help to identify sources of ultra-high energy neutrinos and in consequence the high energy astronomical phenomena which emit cosmic rays for a short $(\sim 100 \mathrm{~s})$ or long $(\sim$ weeks $)$ period of time.

\section{References}

[1] T.K Gaisser and T.Stanev, Astropart. Phys. 39-40 (2012) 120.

[2] K. Koyama et al., Nature 378 (1995) 255.

[3] A.M. Atoyan, C.Dermer, New Astron. Rev. 48 (2004) 381.

[4] A. Neronov, M. Ribordy, Phys. Rev. D 80 (2009) 083008.

[5] A. Mucke et al., Astropart. Phys. 18 (2003) 593.

[6] M.G. Aartsen et al., Science 342 (2013) 1242856.

[7] MAGIC Collaboration: http://http://magic.mppmu.mpg.de/.

[8] VERITAS Collaboration: http://veritas.sao.arizona.edu/.

[9] M. Ackermann et al., Proc. 29th ICRC (2005) [arXiv:astro-ph/0509330].

[10] R. Abbasi et al., Proc. 32th ICRC (2013) [arXiv:1111.2741], M.G. Aartsen et al., Proc. 33th ICRC (2013) [arXiv:1309.6979].

[11] E. Waxman, J. Bahcall, Phys. Rev. Lett., 78 (1997) 2292.

[12] S. Ando, J.F. Beacom, Phys. Rev. Lett., 95 (2005) 061103.

[13] R. Abbasi et al., Astron. Astrophys., A60 (2012) 539.

[14] C.W. Akerlof et al., Pub. of the Astro. Soc. of the Pacific 115 (2003) 132.

[15] N. Law et al., Pub. of the Astro. Soc. of the Pacific 121 (2009) 1395.

[16] N. Gehrels et al., The Astrophys. Journal 611 (2004) 1005.

[17] E.M. Schlegel MNRAS 244 (1990) 269; N. Smith et al., The Astrophys. Journal 143 (2012) 17.

[18] N. Kaiser, Proc. SPIE 5489 (2004) 11; E.A. Magnier et al., The Astrophys. Jour. Supp. 205 (2013) 20.

[19] J. Braun, M. Baker, J. Dumm et al., Astropart. Phys. 33 (2010) 175.

[20] Fermi LAT Second Source Catalog: http://heasarc.gsfc.nasa.gov/W3Browse/fermi/fermilpsc.html.

[21] A. Achterberg et al., Astropart. Phys. 26 (2006) 155.

[22] K. Satalecka et al, for the IceCube Coll., Proc. 30th ICRC (2007) [arXiv:0711.0353].

[23] S. S. Kerthi et al., Neural Comp. 13 (2001) 637.

[24] M.G. Aartsen et al. submitted to Astrophysical Journal (2015) [arXiv:1506.03115].

[25] IceCube Coll., paper 504 these proceedings.

[26] http://www.mpi-hd.mpg.de/hfm/HESS/. 